\title{
Stochastic interactions of rough particles in viscous shear flow
}

\author{
M. Darbeheshti, J. Stokan, U. Dam \& M. S. Ingber \\ Department of Mechanical Engineering, \\ University of Colorado Denver, USA
}

\begin{abstract}
Particle interactions in highly-viscous nonlinear and linear shear flows play an important role in a variety of applications, including composite materials processing, microfluidics, chromatography, and particle resuspension, to name a few. Binary interactions between particles can provide information used in rheological models for suspension flows such as migration rates and selfdiffusivity. In past numerical studies, particle roughness has been treated, for the most part, as a constant, static quantity. In the current study, roughness is treated as a stochastic parameter. Hence, quantities such as dispersion, net particle migration, and self-diffusivity also become stochastic parameters. Numerical simulations are performed using a semi-analytic solution for the motion of two particles in an unbounded flow field to determine the effects of random particle roughness.

Keywords: particle interactions, particle migration, particle dispersion, selfdiffusivity, suspension flows.
\end{abstract}

\section{Introduction}

Interactions of smooth particles in isothermal, Stokes flow are theoretically reversible. However, irreversibility can be introduced in a variety of ways including viscosity perturbations, inter-particle static forces, salvation forces, and particle roughness. In particular, particle roughness in suspension flows has been shown to cause asymmetries in particle trajectories in linear shear flows [1,2], particle pair migration [2] in nonlinear shear flows, and asymmetric pair distribution functions [3] in low-concentration suspension flows.

Experiments performed by Smart and Leighton [4] and Heath et al. [5] have shown that the magnitude of particle roughness can, in certain cases, be determined 
by measuring the difference in the time it takes for a particle to sediment towards a plane through a given distance, inverting the plane, and then measuring the time it takes the particle to sediment the same distance away from the plane. However, in the study by Smart and Leighton, particle roughness was made somewhat uniform by gluing sieved sand particles of relatively uniform size to the outside of larger spheres. In practice, roughness is not very uniform.

Papova et al. [6] performed experiments with three different types of PMMA particles in terms of the surface treatment, namely, original surface (relatively) smooth particles, lapped particles, and bead-blasted particles. They showed in their experiments conducted in a Couette device that particle migration scaled essentially with the square root of the root-mean-square particle roughness.

Ingber et al. [7] performed both experiments and numerical simulations of five particles in a Couette device. In the simulations, a traction-corrected boundary element method was employed with a static surface roughness model. They determined that the increase in the so-called particle radial moment matched in the average over the 52 experiments and corresponding numerical simulations when the imposed surface roughness in the numerical simulations was approximately four times the average physical particle roughness.

For the most part, numerical simulations of binary sphere interactions in shear flow have treated roughness as a static quantity $[2,8,9]$. Zhao and Davis [10] considered the interaction of sedimenting spheres with essentially two levels of surface roughness. In general, surface roughness is a stochastic quantity. In this research, the effects of stochastic surface roughness on quantities such as particle dispersion, particle migration, and particle self-diffusivity is studied.

\section{Numerical methodology}

The problem under consideration is the binary interaction of two rough particles in the zero-Reynolds-number limit. The governing equation for the fluid is the Stokes equation and the governing equations for the particles are the equilibrium and kinematic equations. The numerical method used to perform the simulations in the current research is based on a semi-analytic solution for the motion of two spheres suspended in an unbounded but otherwise arbitrary shear flow [11]. The semi-analytic method is a vast generalization of classical bisphericalcoordinate solutions for two spheres moving along or perpendicular to their line of centers, rotating about the centerline in a quiescent liquid, or suspended in linear shear flow. The method is highly efficient and very convenient since the algorithm does not require any local expansions of the ambient velocity field $\mathbf{u}(\mathbf{x})$, but simply operates with the values of $\mathbf{u}(\mathbf{x})$ in the vicinity of the spheres, which can be calculated either by an analytic formula (e.g., for Poiseuille flow) or by a user-provided routine for more complex cases. The method provides linear and angular velocities as a function of position. Once the linear and angular velocities are determined, the particles are repositioned in space using a third-order, variable-time-step Runge-Kutta routine. The governing equations considered in this research are reversible. Further, this particular numerical 
algorithm is extremely accurate, and hence, the particle motions for presumed smooth spheres are essentially reversible. The roughness model considered in this research is essentially equivalent to the non-locked model of DaCunha and Hinch [8] in which particles are not allowed to have separations less than a specified roughness $\epsilon$, but are allowed to rotate relative to each other. However, unlike previous research $[2,8]$, roughness is presumed to be a random stochastic quantity modeled as a normal distribution with specified average roughness and standard deviation.

\section{Results}

We consider the interaction of two rough spheres in nonlinear shear (Poiseuille) flow. The far-field velocity profile is in the $x$-direction and is given by

$$
u=b-c(z-d)^{2}
$$

where $b, c$, and $d$ are constants. The average sphere radius is denoted by $a$. The following definitions are useful in characterizing these problems. The coordinates of the two particles are given by $\left(x_{1}, y_{1}, z_{1}\right)$ and $\left(x_{2}, y_{2}, z_{2}\right)$. The initial center to center separations of the spheres in the three coordinate directions are given by $\Delta x_{-\infty}, \Delta y_{-\infty}$, and $\Delta z_{-\infty}$. The shear plane is the $x-z$ plane, the far-field velocity is in the $x$-direction, and the initial separation is given by $\Delta x_{-\infty} / a=$ $-10 a$. The simulations are stopped when $x_{2}-x_{1}=10 a$ which then sets the downstream separations denoted by $\Delta x_{\infty}, \Delta y_{\infty}$, and $\Delta z_{\infty}$. The particle trajectories are typically drawn with respect to the transient separation $\Delta x=$ $x_{1}-x_{2}$.

Typical transient trajectories of the particle pairs in the shear plane are shown in Fig. 1 for the case $\Delta z_{-\infty} / a=0.2, \Delta y_{-\infty} / a=0.0, b=41 / 40, c=5 / 8$, $d=1 / 2$, and particle roughness ranging between $\epsilon / a=0$ and $\epsilon / a=0.01$. As seen in the figure for the case $\epsilon / a=0.0$ (smooth spheres), the particle trajectories are symmetric about $\Delta x / a=0$ and, in particular, $\Delta z_{\infty}=\Delta z_{-\infty}$. This indicates that the particle trajectories are essentially reversible. As the roughness increases, the in-plane particle dispersion $\Delta z_{\infty}-\Delta z_{-\infty}=\Delta z_{ \pm \infty}$ is also seen to increase indicating increasing irreversibility in the system with increasing particle roughness. Another quantity of interest is the net particle migration which is the location of the center of mass of the particle pair, $z_{\mathrm{cm}}$, at the end of the simulation. Again, for smooth particles, there is no net particle migration. As the particle roughness increases, the net particle migration also increases with $z_{c m}>0$ indicating that the particle pair has migrated towards the low-shear-rate region of the flow field.

The analysis of in-plane particle dispersion and migration in previous studies $[2,11]$ has assumed that the particle roughness is constant. The effect of stochastic surface roughness on particle dispersion and migration over 20 simulations is shown in Fig. 2 again for the case $\Delta z_{-\infty}=0.2, \Delta y_{-\infty}=0.0, b=41 / 40$, $c=5 / 8$, and $d=1 / 2$. As seen in the figure, both particle dispersion and migration 


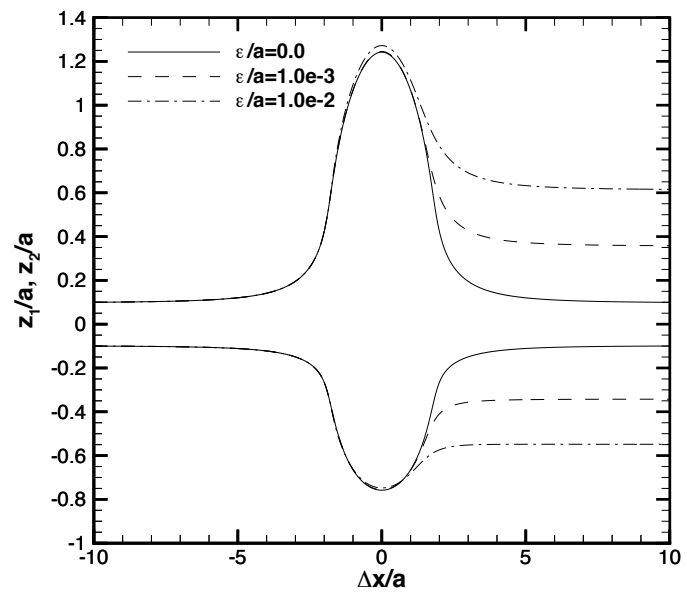

Figure 1: Particle trajectories in the shear plane of two rough spheres suspended in Poiseuille flow with initial positions $(-5.0,-0.05,0.1)$ and $(5.0,0.05,-0.1)$, $\Delta y_{-\infty}=0.0$ and $\Delta z_{-\infty}=0.2$.

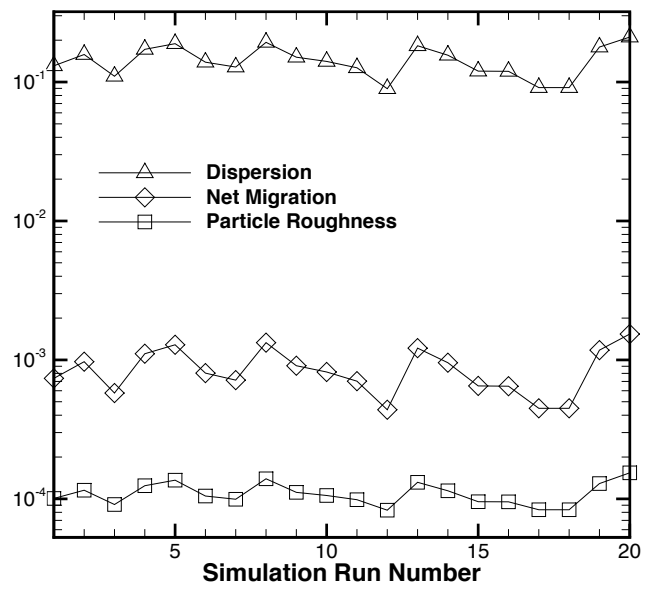

Figure 2: Dispersion, migration, and particle roughness for 20 simulations for an average roughness of $\epsilon / a=1.0 \mathrm{e}-4$ and standard deviation of $\sigma=2.0 \mathrm{e}-5$. 


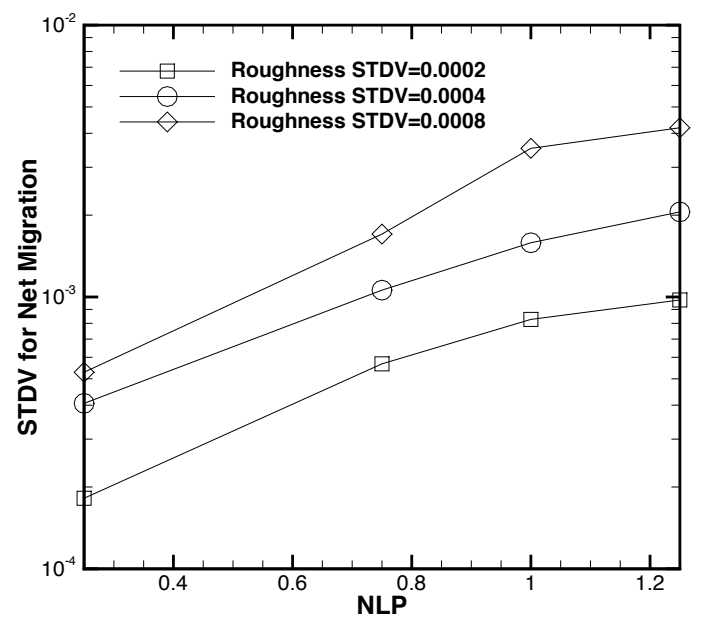

Figure 3: Standard deviation, STDV, of the particle net migration as a function of the nonlinearity parameter, $N L P$, for the case $\Delta z_{-\infty} / a=0.2$, $\Delta y_{-\infty}=0.0$, and $\epsilon / a=1.0 \mathrm{e}-3$.

correlate essentially perfectly with the stochastic particle roughness which is not surprising considering roughness is the only form of irreversibility introduced into the flow. (There is some very small irreversibility introduced into the simulations caused by numerical error, but this irreversibility is imperceptible in the figure.)

A quantity of interest is the so-called nonlinearity parameter, $N L P$, defined by

$$
N L P=\frac{a \nabla \dot{\gamma}}{\dot{\gamma}+\dot{\gamma}_{N L}}
$$

where $a$ is the sphere radius, $\dot{\gamma}$ is the local shear rate calculated at the initial location of the center of mass of the particle pair, and $\dot{\gamma}_{N L}$ is the so-called nonlocal contribution to the shear rate ( [12]). The nonlocal shear rate is given by

$$
\dot{\gamma}_{N L}=\frac{a}{D} \dot{\gamma}_{o}
$$

where $D$ is distance between the walls and $\dot{\gamma}_{o}$ is the shear rate for the unperturbed flow at the wall. As the name suggests, the nonlinearity parameter is a measure of the nonlinearity of the flow field. The effect of the nonlinearity parameter on the standard deviation of the net migration for initial separation given by $\Delta z_{-\infty} / a=$ $0.2, \Delta y_{-\infty}=0.0$, and average sphere roughness given by $\epsilon / a=0.001$ is shown in Fig. 3 for three different values of roughness standard deviation. As seen in the figure, the standard deviation of the net migration increases with both 


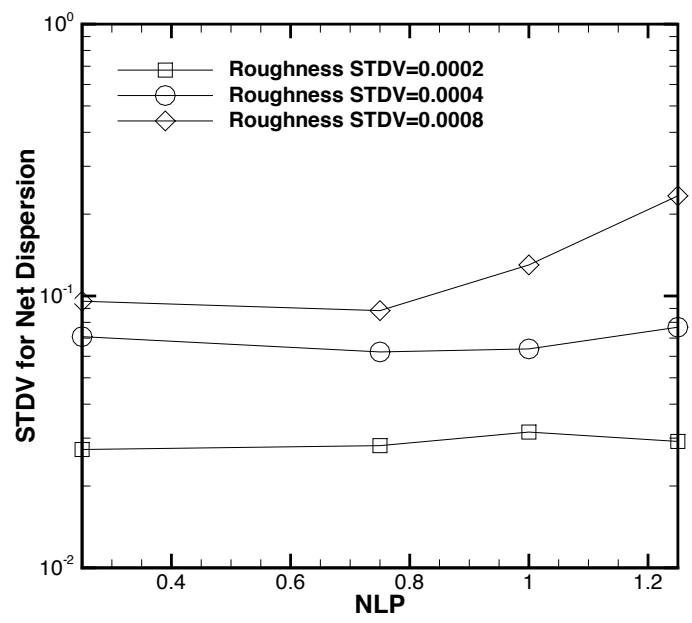

Figure 4: Standard deviation, STDV, of the particle dispersion as a function of the nonlinearity parameter, $N L P$, for the case $\Delta z_{-\infty} / a=0.2, \Delta y_{-\infty}=$ 0.0 , and $\epsilon / a=1.0 \mathrm{e}-3$.

increasing nonlinearity in the flow field and increasing standard deviation of the sphere roughness.

The dispersion, $\Delta z_{ \pm \infty}$, as a function of the nonlinearity parameter is shown in Fig. 4. As seen in the figure, for the two lower values of the roughness standard deviation, there is little influence of the nonlinearity parameter on the dispersion. This is actually an expected result as it has previous been shown $[2,11]$ that the nonlinearity parameter has little influence on particle self-diffusivity which is primarily a function of particle dispersion as discussed below.

The final quantity considered in this research is the in-plane self-diffusivity of binary sphere iterations in linear shear flow. The self-diffusivity in the shear plane, $D_{z}^{s}$, can be calculated by evaluating the following integral ( [2])

$$
D_{z}^{s}=\phi a^{2} \dot{\gamma} \frac{3}{8 \pi} \int_{-\infty}^{\infty} \int_{-\infty}^{\infty}\left(\Delta z_{ \pm \infty}\right)^{2} \Delta z_{-\infty} d y_{-\infty} d z_{-\infty}
$$

The in-plane self-diffusivity $D_{z}^{s}$ for a linear shear flow over 20 different simulations with average particle roughness of $\epsilon / a=0.001$ is shown in Fig. 5 for three different standard deviations of the roughness. As seen in the figure, the average in-plane self-diffusivity does not change appreciably with roughness standard deviation. In fact, the average self-diffusivity, $\bar{D}_{z}^{s}$, is given by $7.91 \mathrm{e}-4$, $7.82 \mathrm{e}-4,7.86 \mathrm{e}-4$ for roughness standard deviations of $1.0 \mathrm{e}-2,1.0 \mathrm{e}-4$, and $1.0 \mathrm{e}-6$, respectively. Similarly, the standard deviation of the in-plane self-diffusivity is 


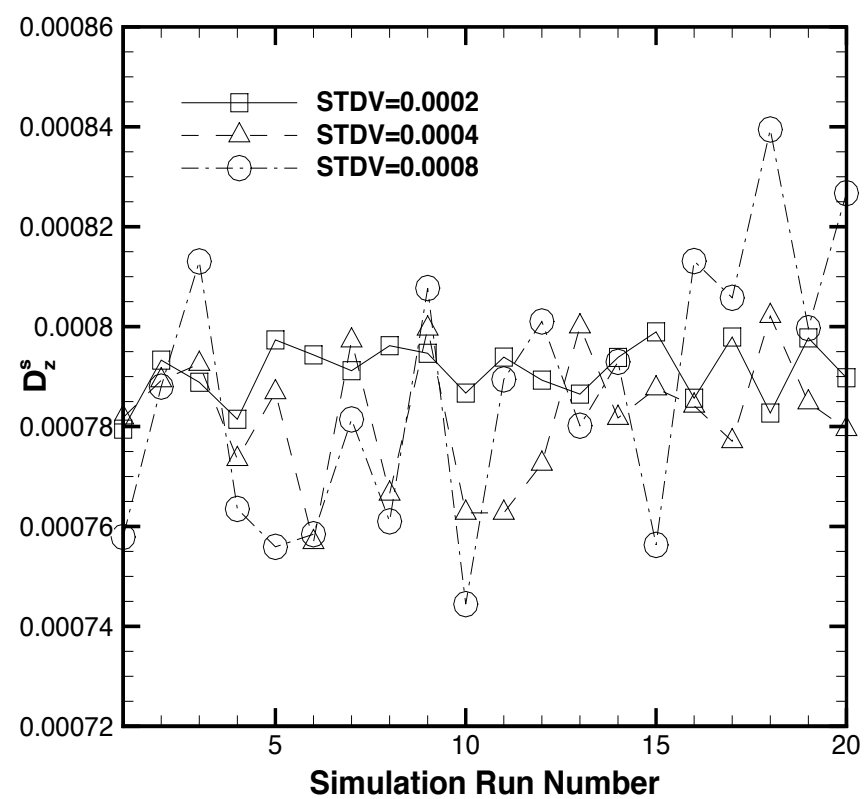

Figure 5: In-plane self-diffusivity $D_{z}^{s}$ for a linear shear flow.

given by $5.86 \mathrm{e}-6,1.32 \mathrm{e}-5$, and $2.67 \mathrm{e}-5$ again for roughness standard deviations of $1.0 \mathrm{e}-2,1.0 \mathrm{e}-4$, and $1.0 \mathrm{e}-6$, respectively. That is, the standard deviation of the self-diffusivity essentially doubles with a doubling of the standard deviation of the roughness over the range considered in this study.

\section{Conclusions}

The effects of stochastic surface roughness on the binary interactions of spheres in linear and nonlinear shear flow are considered in this research. Stochastic surface roughness causes parameters such as particle dispersion, migration, and self-diffusivity to also be stochastic parameters. There is essentially a perfect correlation between increases and decreases in the surface roughness with increases and decreases in particle-pair dispersion and net migration. An increase in the nonlinearity parameter caused an increase in the standard deviation of the net particle migration, but had little effect on the standard deviation of the net particle dispersion. The standard deviation of particle roughness had essentially no effect on the average value for the in-plane self-diffusivity. However, the standard deviation of the self-diffusivity scales linearly with slope one with the standard deviation of the particle roughness over the range of surface roughnesses considered in this study. 


\section{Acknowledgement}

This material is based upon work supported by the National Science Foundation under Grant No. CBET-1335781. Any opinions, findings, and conclusions or recommendations expressed in this material are those of the authors and do not necessarily reflect the views of the National Science Foundation.

\section{References}

[1] Zeng, S.L., Kerns, E.T. \& Davis, R.H., The nature of particle contacts in sedimentation. Phys Fluids, 8(6), pp. 1389-1396, 1996.

[2] Ingber, M.S., Feng, S., Graham, A.L. \& Brenner, H., The analysis of selfdiffusion and migration of rough spheres in nonlinear shear flow using a traction-corrected boundary element method. J Fluid Mech, 598, pp. 267292, 2008.

[3] Rampall, I., Smart, J.R. \& Leighton, D.T., The influence of surface roughness on the particle-pair distribution function of dilute suspensions of noncolloidal spheres in simple shear flow. J Fluid Mech, 339, pp. 1-24, 1997.

[4] Smart, J.R. \& Leighton, D.T., Measurement of the hydrodynamic surface roughness of noncolloidal spheres. Phys Fluids A, 1, pp. 52-60, 1989.

[5] Heath, C.E., Feng, S., Day, J.P., Graham, A.L. \& Ingber, M.S., Near Contact Interactions Between a Sphere and a Plane. Phys Rev E, 77, pp. 026307-1026307-10, 2008.

[6] Popova, M., Vorobieff, P. \& Graham, M.S.I.A.L., Interaction of two particles in a shear flow. Phys Rev E, 75, pp. 066309-5, 2007.

[7] Ingber, M.S., Mammoli, A.A., Vorobieff, P., McCollum, T. \& Graham, A.L., Experimental and numerical analysis of irreversibilities particles suspended in a Couette device. J Rheol, 50(2), pp. 99-114, 2006.

[8] DaCunha, F.R. \& Hinch, E.J., Shear-induced dispersion in a dilute suspension of rough spheres. J Fluid Mech, 309, pp. 211-223, 1996.

[9] Wilson, H.J. \& Davis, R.H., The viscosity of a dilute suspension of rough spheres. J Fluid Mech, 421, pp. 339-367, 2000.

[10] Zhao, Y. \& Davis, R.H., Interaction of sedimenting spheres with multiple surface roughness scales. J Fluid Mech, 492, pp. 101-2998, 2003.

[11] Ingber, M.S. \& Zinchenko, A., Semi-analytic solution of the motion of two spheres in arbitrary shear flow. J Mult Flow, 42, pp. 152-163, 2012.

[12] Miller, R.M. \& Morris, J.F., Normal stress-driven migration and axial development in pressure-driven flow of concentrated suspensions. J NonNewt Fluid Mech, 135, pp. 149-165, 2006. 